\title{
Coupling between the ocean and an ice shelf
}

\author{
Colin Fox \\ Department of Mathematics and Statistics, University of Auckland, Private Bag, \\ Auckland, New Zealand \\ VERnON A. SQUiRE \\ Department of Mathematics and Statistics, University of Otago, P.O. Box 56, \\ Dunedin, New Zealand
}

\begin{abstract}
The possibility of long-period ocean waves coupling to an ice shelf is investigated. A thick elastic plate model is used for the ice shelf with comparisons made to the simpler thin-plate model. The strain set up on the ice shelf by a normally incident single frequency ocean wave is calculated by completely solving the equations governing the velocity potential for such a system. In the absence of measurements on an ice shelf, existing measurements of long-period strain on an ice tongue are used to estimate the required incident amplitude in the open water to induce the observed oscillations. It is found that the height of seas required indicates that ocean wave driving is a plausible forcing mechanism for observed oscillations.
\end{abstract}

\section{INTRODUCTION}

Several observational studies suggest that long-period oscillations occur in ice shelves and ice tongues at periods well beyond those of the typical open ocean swell; for example, long-period oscillations were observed on an ice shelf during the " $90^{\circ}$ South" expedition (personal communication from M. Kristensen). Oscillations with 40-50 s period, for example, have been measured in the Erebus Glacier Tongue, McMurdo Sound, Antarctica. Further, there is some evidence to suggest that the waves are propagating (personal communication from W.H. Robinson), i.e. that they are not entirely due to free oscillations set up by some applied action which may only exist for a short time and is aperiodic. The questions which then remain are where does the energy come from to drive these travelling waves, and how efficient is the coupling between the ocean and the ice shelf/tongue? In this paper we will examine the possibility of long-period surface gravity waves coupling between the ocean and one of those geometries, namely a massive ice shelf.

During the summer months in particular, meltwater from the ice shelf may overlie the denser sea water leading to a stratified water column and the possibility of internal gravity waves. For a given wave period, there are two travelling internal waves, one corresponding to an homogeneous water body with the average density and another in which the surface and internal waves are in antiphase. The wavelength of the latter type of internal wave is usually much less than the wavelength of the former. For example, for the periods and geometries that we will consider in this paper, the wavelengths of the first type of wave are in the range 1 to $10 \mathrm{~km}$, whereas the wavelength of the second type is less than $10 \mathrm{~m}$, even with extreme stratification. Coupling to an ice shelf at such short wavelengths is unlikely to be significant. Hence, the short internal waves can be neglected as a forcing mechanism or, equivalently, the ocean can be regarded as being homogeneous.

The coupling question is investigated in this paper by using a thick elastic plate equation to study the transmission of wave energy into an ice shelf. The complete set of wave potentials is used, viz. travelling plus an infinite sum of evanescent waves in the open water, and travelling, damped-travelling, plus an infinite sum of evanescent waves in the ice-covered region. Unlike earlier approximate solutions, which omit the evanescent contribution (see Fox and Squire, 1990, for references), this ensures continuity of the potential and the velocity over the depth, thereby solving the mathematical problem completely. Hence, the amplitude reflexion and transmission coefficients can be found to any desired precision. Further, the strain on the ice shelf induced by ocean waves or swell can be calculated from the velocity potential, once found.

By working backwards, the incident wave heights in the open water, required to induce flexural oscillations in the correct frequency band with the observed magnitude on the ice shelf, will then be found. There is little energy available in the open sea at the observed periods, and it is of interest to discover from where the input derives. Swells at such long periods are unlikely, for example, unless ice shelves and tongues react selectively to a band of long wave periods. This is an interesting possibility, as it suggests that existing accelerometer-type instrumentation (as typically used in wave buoys) has in- 
sufficient resolution to detect such low frequency waves, whereas the band-pass filtering action of an ice shelf, combined with alternative curvature-based measurement techniques, makes detection possible.

Seiches, and harmonics of seiches, are other possible forcing mechanisms, as are nonlinear modulation, surf beats, edge waves, or wave packet formation, although these seem rather esoteric mechanisms.

\section{MATHEMATICAL MODEL}

We investigate the propagation of ocean waves into an ice shelf by solving the following mathematical model of the physical system. We will assume that the ocean is homogeneous and has constant depth, the incoming ocean waves have small amplitude and propagate normal to the edge of a massive (half plane) ice shelf of constant thickness. This model has no variation parallel to the edge of the ice shelf, and so is essentially twodimensional. In cases where the thickness of the ice shelf is a small proportion of the ocean's depth, we can think of the ice shelf as floating on the water. This geometry is shown in Figure 1.

Using the general linear theory for small amplitude waves, along with the usual assumption of irrotational flow, we define a velocity potential within the water, $\Phi(x, y, t)$, which satisfies Laplace's equation at each instant in time. Initially, we will consider a single monochromatic ocean wave with time dependence $e^{i \omega t}$ where the period of the wave is $T=2 \pi / \omega$. As our model is linear, and invariant in time, it follows that $\Phi(x, y, t)=\phi(x, y) e^{i \omega t}$ where the spatial dependence also satisfies Laplace's equation; i.e.,

$\frac{\partial^{2} \phi}{\partial x^{2}}+\frac{\partial^{2} \phi}{\partial y^{2}}=0$, for $-\infty<x<\infty$ and $0<y<H$.

At the sea floor, the vertical component of velocity must vanish leading to the boundary condition

$$
\frac{\partial \phi}{\partial y}=0, \quad \text { for } \quad-\infty<x<\infty \quad \text { and } \quad y=0 .
$$

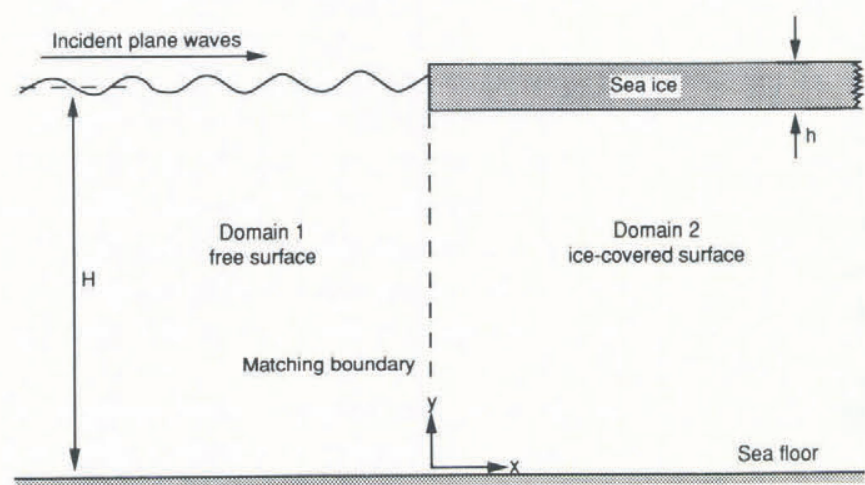

Fig. 1. Schematic showing the semi-infinite sheet of sea ice of thickness $h$ floating on water of depth H. Surface gravity waves travel from left to right and are reflected and transmitted at the sea-ice boundary. Note that the $y$-axis is vertically upwards with $y=0$ at the sea floor and $y=H$ at the surface; this is done to simplify subsequent algebra.
A boundary condition for the surface of the open sea can be established using the kinematic condition and Bernoulli's theorem (Phillips, 1977) which in terms of $\phi$ is

$$
g \frac{\partial \phi}{\partial y}=\omega^{2} \phi, \quad \text { for } \quad-\infty<x<0 \quad \text { and } \quad y=H,
$$

where $g$ is the acceleration due to gravity.

In the absence of viscous and other nonlinear terms, Equations (1) to (3) are the general equations governing the potential within an homogencous constant-depth sea with a free surface. In this paper, we are concerned with long-period waves and later we will find that only small amplitudes are involved. Consequently, the nonlinear terms in a more complete formulation are negligible.

\section{Thick-plate model of the ice shelf}

The propertics of the ice shelf will be modelled as a thick isotropic elastic plate. The most complete work to date on the problem of ocean coupling to floating ice sheets has modelled the ice as a thin elastic plate (Evans and Davies, 1968; Fox and Squire, 1990, 1991; Squire and Fox, 1990). The thin-plate models neglect the effects of the finite thickness of the plate and, hence, neglect its rotary inertia and transverse shear deformation. An equation relating the transverse displacement, $w$, of a thick plate to the applied normal pressure, $p$, has been derived by Mindlin (1951) from the three-dimensional equations including the effects of rotary inertia and transverse shear with suitable assumptions such as the plate faces remaining parallel. This relation is

$$
\begin{gathered}
\left(\nabla^{2}-\frac{\rho_{\mathrm{i}}}{\mu G} \frac{\partial^{2}}{\partial t^{2}}\left(L \nabla^{2}-\frac{\rho_{\mathrm{i}} h^{3}}{12} \frac{\partial^{2}}{\partial t^{2}}\right) w+\rho_{\mathrm{i}} h \frac{\partial^{2} w}{\partial t^{2}}\right. \\
=\left(1-\frac{L}{\mu G h} \nabla^{2}+\frac{\rho_{\mathrm{i}} h^{3}}{12 \mu G} \frac{\partial^{2}}{\partial t^{2}}\right) p,
\end{gathered}
$$

where $\rho_{\mathrm{i}}$ is the density of the ice shelf, $h$ is the thickness of the ice shelf, $\mu$ is a shear constant which is close to unity and, for a Young's modulus $E$ and Poisson's ratio $\nu, G=E / 2(1+\nu)$ is the shear modulus and $L=E h^{3} / 12\left(1-\nu^{2}\right)$ is the flexural rigidity of the plate. In order to determine the motion of the plate uniquely, boundary conditions must be applied at its edges. The natural boundary conditions can be found by comparing Equation (4) with the variational principle that the plate must satisfy (Hildebrand, 1965), which can in fact be derived from the differential equation. The two boundary conditions found this way are

$$
\begin{gathered}
\left(L\left[\nabla^{2}+(1-\nu) \frac{\partial^{2}}{\partial s^{2}}\right]-\rho_{\mathrm{i}}\left[\frac{L}{\mu G}+\frac{h^{3}}{12}\right] \frac{\partial^{2}}{\partial t^{2}}\right) \frac{\partial w}{\partial n}=0 \\
\text { and } \quad L\left(\frac{\partial^{2} w}{\partial n^{2}}+\nu \frac{\partial^{2} w}{\partial s^{2}}\right)=0
\end{gathered}
$$

where $\partial / \partial n$ and $\partial / \partial s$ denote differentiation normal and parallel to the boundary, respectively, and the Laplacian acts in the plane of the plate. 
When the plate thickness, $h$, is small and when we are only concerned with waves with a long period, $T$, the terms in Equation (4) representing rotary inertia and transverse shear can be neglected, leaving the simpler thin-plate equation

$$
L \nabla^{4} w+\rho_{\mathrm{i}} h \frac{\partial^{2} w}{\partial t^{2}}=p .
$$

The boundary conditions for this simpler case are given by neglecting the time derivative in Equation (5) and by Equation (6) as it stands. The case of ocean wave coupling to thin ice plates has been solved in detail by Fox and Squire $(1990,1991)$ and Squire and Fox (1990).

\section{Restriction of the thick-plate equations to the model}

Assuming no cavitation between the ice and water, the thick-plate equation can be combined with the dynamic and kinematic conditions at the surface of the water to give a boundary condition for $\phi$ at that surface. When restricted to the two-dimensional monochromatic model, that condition can be written

$$
\begin{array}{r}
\left(L \frac{\partial^{4}}{\partial x^{4}}+A \frac{\partial^{2}}{\partial x^{2}}+B\right) \frac{\partial \phi}{\partial y}=-\left(C \frac{\partial^{2}}{\partial x^{2}}+D\right) \phi, \\
\text { for } 0<x<\infty \text { and } y=H,
\end{array}
$$

where for brevity we have defined the constants

$$
\begin{aligned}
& A=\frac{L}{\mu G h}\left(m \omega^{2}-\rho_{\mathrm{w}} g\right)+\frac{\omega^{2} m h^{2}}{12}, \\
& B=\left(m \omega^{2}-\rho_{\mathrm{w}} g\right)\left(\frac{\omega^{2} m h}{12 \mu G}-1\right), \\
& C=\frac{\rho_{\mathrm{w}} \omega^{2} L}{\mu G h} \text { and } \\
& D=\rho_{\mathrm{w}} \omega^{2}\left(\frac{\omega^{2} m h}{12 \mu G}-1\right),
\end{aligned}
$$

with $\rho_{\mathrm{w}}$ denoting the density of sea water and $m=\rho_{\mathrm{i}} h$ being the mass per unit area of the ice shelf. As an example of the relative magnitudes of the constants, for $t=50 \mathrm{~s}, H=500 \mathrm{~m}$ and $h=200 \mathrm{~m}$, we find $L=4.4 \times$ $10^{15}, A=-6.4 \times 10^{7}, B=7.1 \times 10^{3}, C=1.7 \times 10^{5}$ and $D=-16$. When the boundary conditions (Equations (5) and (6)) are similarly restricted they become

$$
\begin{array}{rlrl}
L \frac{\partial^{3}}{\partial x^{3}} \frac{\partial \phi}{\partial y}+m \omega^{2}\left(\frac{L}{\mu G h}+\frac{h^{2}}{12}\right) \frac{\partial}{\partial x} \frac{\partial \phi}{\partial y} & =0 \\
L \frac{\partial^{2}}{\partial x^{2}} \frac{\partial \phi}{\partial y}=0 & \text { at } x & =0^{+} \text {and } y=H, \\
& \text { at } x & =0^{+} \text {and } y & =H .
\end{array}
$$

Equations (1), (2), (3), (8), (9) and (10) give a full statement of the mathematical model.

\section{Modes of the system}

A complete set of potentials can be found, each satisfying the differential Equation (1) and the bottom boundary condition (Equation (2)) and one of the surface conditions (Equations (3) and (8)), by separating variables on either side of the line $x=0,0<y<H$. These po- tentials are the modes of the system and any solution of the model can be represented as a combination of these modes. Each mode has the form $\phi=e^{k x} e^{ \pm i k y}$. Pairs of these modes can be combined to give the equivalent set of modes

$$
\phi=e^{k x} \sin (k y) \quad \text { and } \quad \phi=e^{k x} \cos (k y) .
$$

The boundary condition at the sea floor rules out the modes with $\sin (k y) y$-dependence, leaving only those with $\cos (k y) y$-dependence. Only certain values of $k$ give rise to modes which also satisfy one of the boundary conditions at the sea surface.

In the region $x<0$ the modes must satisfy the open sea condition (Equation(3)) which demands that $k$ satisfies the dispersion equation

$$
k \tan (k H)=-\frac{\omega^{2}}{g} .
$$

This equation has two pure imaginary roots $\pm i k_{\mathrm{T}}\left(k_{\mathrm{T}}>\right.$ 0 ), corresponding to travelling waves propagating in the positive and negative $x$ directions, and infinitely many real roots $\pm k_{n}(n=1,2, \ldots)$, where $\left(n-\frac{1}{2}\right) \pi / H<k_{n}<$ $n \pi / H$, corresponding to evanescent modes.

In the ice-covered region, $x>0$, we will write the modes as $\phi=e^{\kappa x} \cos (\kappa y)$, replacing $k$ by $\kappa$ to distinguish between the two regions. The wavenumber $\kappa$ must satisfy the more complicated dispersion equation

$$
\kappa \tan \kappa H=\frac{C \kappa^{2}+D}{L \kappa^{4}+A \kappa^{2}+B} .
$$

For physically relevant geometries and most periods this dispersion equation also has two imaginary roots $\pm i \kappa_{\mathrm{T}}\left(\kappa_{\mathrm{T}}>0\right)$ corresponding to travelling waves, four complex roots occurring as plus and minus a complex conjugate pair $\pm \kappa_{\mathrm{D}}$ and $\pm \kappa_{\mathrm{D}}^{*}\left(\kappa_{\mathrm{D}}\right.$ has positive real and imaginary parts) corresponding to damped travelling waves, and infinitely many real roots $\pm \kappa_{n}(n=1,2, \ldots)$, where in general $(n-1 / 2) \pi / H<\kappa_{n}<(n+1 / 2) \pi / H$, each giving an evanescent mode.

The evanescent modes have no phase term and, hence, carry no energy but are vital to the coupling of energy between the open sea and the ice shelf. Because of their $e^{k x}$ ( $e^{\kappa x}$ for the modes beneath the ice) $x$-dependence, the physically relevant evanescent modes decay away from the ice edge. For large magnitudes of $x$, only the travelling waves are present, whereas near the ice edge the evanescent modes have significant amplitude. Thus, in the stationary case that we are considering, of a single period wave incident on an ice shelf, each evanescent mode traps a fixed amount of energy near the boundary between the ice shelf and the open sea. Each evanescent mode does not propagate any energy, but facilitates the transfer of energy from the incident wave to the reflected and transmitted waves.

We will consider only the roots of the dispersion equations giving rise to bounded modes and relevant to the problem of an ocean wave being reflected from, and transmitted into, the ice covered region. In particular, we do not allow any wave propagating from large positive 
$x$ assuming that the transmitted wave is dissipated at, or before, the end of the ice shelf so that no significant wave is reflected there. We may use the relevant modes to write any bounded potential in the open sea region, $x<0$, as

$$
\phi_{\mathrm{o}}=\left(I e^{-i k_{\mathrm{T}} x}+R e^{i k_{\mathrm{T}} x}\right) \cosh k_{\mathrm{T}} y+\sum_{n=1}^{\infty} a_{n} e^{k_{n} x} \cos k_{n} y,
$$

where $I$ and $R$ are the complex coefficients of the incident and reflected travelling waves, respectively, and $\left\{a_{n}\right\}$ is the set of coefficients of the bounded evanescent modes. Similarly, any relevant potential in the ice covered region, $x>0$, can be written

$$
\begin{aligned}
\phi_{\mathrm{i}} & =T e^{i \kappa_{\mathrm{T}} x} \cosh \kappa_{\mathrm{T}} y+b+e^{-\kappa_{\mathrm{D}} x} \cos \kappa_{\mathrm{D}} y \\
& +b_{-} e^{-\kappa_{\mathrm{D}}^{*} x} \cos \kappa_{\mathrm{D}}^{*} y+\sum_{n=1}^{\infty} b_{n} e^{-\kappa_{n} x} \cos \kappa_{n} y,
\end{aligned}
$$

where $T, b_{+}, b_{-}$and $\left\{b_{n}\right\}$ are the complex coefficients of the transmitted wave, the damped travelling waves propagating in the positive and negative $x$ directions, and the infinity of evanescent modes, respectively. The potential in this region must also satisfy the ice-edge conditions (Equations (9) and (10)).

In reality, an ice sheet is not of infinite extent and the assumption that no energy is reflected from its far end may be questioned as the travelling wave suffers no attenuation in our model. With perfect reflection it would be a simple matter to include an additional term in Equation (14) representing the returning wave. An alternative approach, due to Gui and Squire (1989), assumes the ice to behave as a fixed-free beam and solves for the normal modes of the system when the beam is acted upon by a (white) wind pressure spectrum or by a realistic ocean wave spectrum.

\section{Method of solution}

A non-trivial solution is found by specifying unit incident amplitude and then matching the potentials $\phi_{\mathrm{o}}$ and $\phi_{\mathrm{i}}$ on the line $x=0$. The natural quantities to equate are the potential and its derivative normal to the matching boundary, i.e. $\partial \phi / \partial x$. This can be achieved by minimising the error integral

$$
\mathcal{E}=\int_{0}^{H}\left|\phi_{\mathrm{i}}-\phi_{\mathrm{o}}\right|^{2} \mathrm{~d} y+\alpha \int_{0}^{H}\left|\frac{\partial \phi_{\mathrm{i}}}{\partial x}-\frac{\partial \phi_{\mathrm{o}}}{\partial x}\right|^{2} \mathrm{~d} y,
$$

evaluated at $x=0$. The constant $\alpha$ is a weighting term included to improve convergence which we set empirically to 10 in most cases. The solution is thus found by minimising $\mathcal{E}$, subject to the conditions of unit incident amplitude and the boundary conditions at the ice edge given in Equations (9) and (10). The first of these conditions restricts $\phi_{0}$, so that $I=1$, whereas the second and third give rise to two linear equations relating the coefficients of $\phi_{\mathrm{i}}$ in Equation (14). In order to make the calculations possible, the infinite sums in Equations (13) and (14) are terminated at some finite $N$; that is, only the first $N$ evanescent modes are included. The matching is performed for a sequence of increasing $N$ until the solution has converged to its final value. An indepen- dent check is made on the resulting potentials to ensure that they represent a system in energy balance (Fox and Squire, 1990). Using the property that the error, $\mathcal{E}$, is a quadratic form in the real and imaginary parts of the coefficients $I, R,\left\{a_{n}\right\}, T, b_{+}, b_{-}$and $\left\{b_{n}\right\}$, the minimisation is performed, with respect to these coefficients, using a preconditioned linear conjugate gradient algorithm (Gill and others, 1981) restricted to the sub-space in which the three extra conditions are satisfied.

\section{NUMERICAL RESULTS}

The geometries and constants

The following results are based on two water depths, $H$, of $1000 \mathrm{~m}$ and $500 \mathrm{~m}$, and an ice-shelf thickness, $h$, of $200 \mathrm{~m}$. The shelf thickness was chosen to approximate the average dimension of the Erebus Glacier Tongue which tapers from about $300 \mathrm{~m}$ at its hinge line to near $100 \mathrm{~m}$ at its terminus (Holdsworth and Holdsworth, 1978). The $500 \mathrm{~m}$ water depth was chosen to give a shallow water geometry analogous to the water depth in Erebus Bay, beneath the glacier tongue, which is seldom more than $400 \mathrm{~m}$.

The density of sea water is taken to be $\rho_{\mathrm{w}}=$ $1025 \mathrm{~kg} \mathrm{~m}^{-3}$. Constants relating to the physical properties of the ice shelf are as follows. The Young's modulus is $E=6 \times 10^{9} \mathrm{~N} \mathrm{~m}^{-2}$, Poisson's ratio is $\nu=0.3$ and the density of the ice is taken to be $\rho_{\mathrm{i}}=922.5 \mathrm{~kg} \mathrm{~m}^{-3}$. The value of the shear constant $\mu$ is chosen to ensure that the velocity of plane waves predicted by the thick-plate equation corresponds to the equivalent velocity predicted by the full three-dimensional theory (Mindlin, 1951). This implies that $\mu$ satisfies the relations

$$
\begin{array}{r}
\sqrt{\left(1-\frac{1-2 \nu}{2(1-\nu)} \mu^{2}\right)\left(1-\mu^{2}\right)}=\left(1-\frac{1}{2} \mu^{2}\right)^{2} \\
\text { and } 0<\mu<1 .
\end{array}
$$

For the value of $\nu$ above we find that $\mu=0.9274$.

\section{Comparison of the thick-plate and thin-plate models}

It was stated earlier that the thick-plate equation reduces to the simpler thin-plate equation in the thin ice and long wave period limits. The ranges of thickness and period over which this simplifying assumption can be made can be determined by comparing the roots of the dispersion equations resulting from use of the two plate equations. We will compare these formulations in terms of the travelling wavelengths for the geometry given above, over a range of periods.

The travelling wavelengths for the ice-covered region and the region of open sea, given by $2 \pi / \kappa_{\mathrm{T}}$ and $2 \pi / k_{\mathrm{T}}$, respectively, are shown in Figure 2 for wave periods from 1 to $60 \mathrm{~s}$. Both the thick- and thin-plate wavelengths are shown and it can be seen that these coincide for long periods and diverge for periods less than, say, $10 \mathrm{~s}$. In each case of thick-plate, thin-plate and open sea, the wavelengths for the $500 \mathrm{~m}$ depth are less than the wavelengths for $1000 \mathrm{~m}$ water depth at the same period. This is most easily seen for periods above $20 \mathrm{~s}$. It is interesting to note that whereas $1000 \mathrm{~m}$ water is effectively deep 


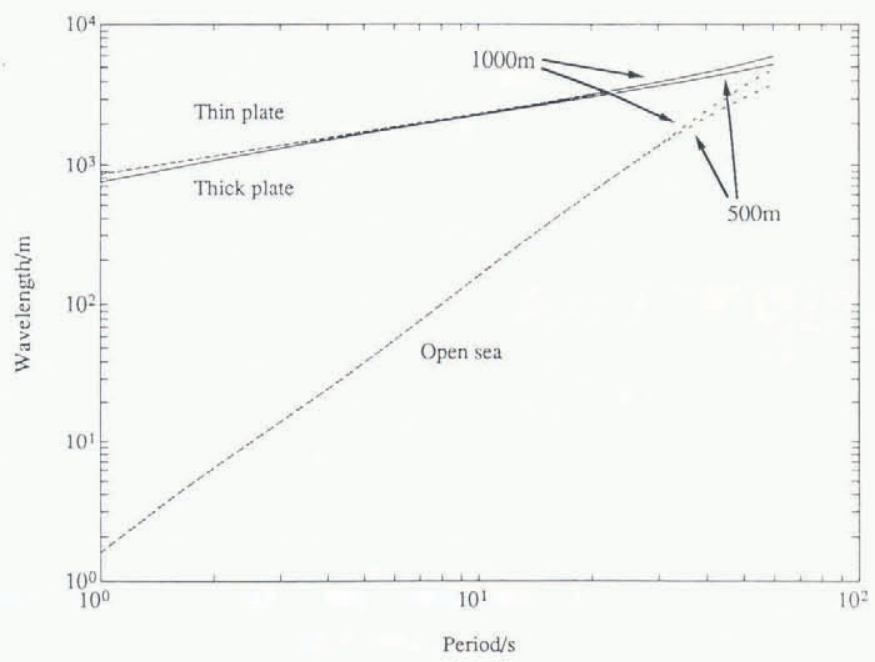

Fig. 2. Wavelengths in metres plotted against period for travelling waves in the open sea and ice-covered region as given by both the thick- and thin-plate models. Two geometrics are represented, $200 \mathrm{~m}$ thick ice on water of $1000 \mathrm{~m}$ and $500 \mathrm{~m}$ depth. The wavelengths for the two depths coincide for short-period waves and are longer for the greater depth at long periods.

with respect to the ocean wave for periods less than $20 \mathrm{~s}$, $1000 \mathrm{~m}$ of water is deep with respect to the ice-coupled wave only for the smallest period shown. Also note that $500 \mathrm{~m}$ of water is not deep with respect to the ice-coupled wave for any period shown and is shallow for all waves of period $40 \mathrm{~s}$ and above. It is also noteworthy, and perhaps contrary to expectation, that the thick-plate model gives a wavelength closer to the open-sea wavelength than does the thin-plate model. This suggests that the thick plate is softer than the thin plate, and the effect may be due to the greater number of degrees of freedom associated with the thick plate (personal communication from K. Hutter).

The ratio of travelling wavenumbers for the thick- and thin-plate models is plotted in Figure 3 for $1000 \mathrm{~m}$ water depth and this shows more clearly the agreement and divergence of the two values. The analogous curve for $500 \mathrm{~m}$ water depth is essentially identical. Note that for periods above $10 \mathrm{~s}$ the two values differ by less than $2 \%$, falling to less than $1 \%$ difference for periods above $20 \mathrm{~s}$. The difference rises to $10 \%$ for periods less than $2 \mathrm{~s}$, but for such short periods both models will predict the total reflection of an incoming wave.

A similar pattern of percentage difference, between the roots of the thin- and thick-plate dispersion equations, occurs for the complex root $\kappa_{\mathrm{D}}$ and the first real root $\kappa_{1}$. The second real root, $\kappa_{2}$, shows less dependence on which equation is used and the third and subsequent real roots $\left(\kappa_{n}, n=3,4, \ldots\right)$ are essentially equal for the two equations over the entire range of periods. Thus, we expect that the simpler thin-plate model should correctly predict the thick-plate behaviour for periods of $20 \mathrm{~s}$ and above.

More usual as a measure of relative plate thickness is the ratio of plate thickness to the wavelength of the

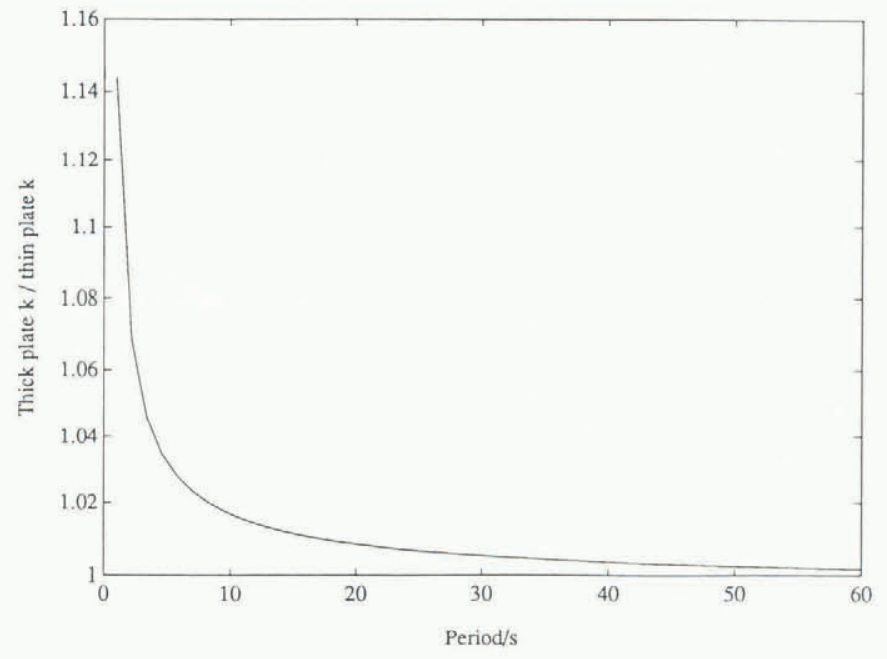

Fig. 3. Ratio of ice-coupled travelling wavelengths as predicted by the thick-plate and thin-plate models plotted against period for $200 \mathrm{~m}$ thick ice on water of $1000 \mathrm{~m}$ depth. The analogous curve for $500 \mathrm{~m}$ water depth is essentially identical.

travelling wave. This ratio is shown in Figure 4 for the same range of periods used above and for both water depths. For periods below approximately $8 \mathrm{~s}$ the plate thickness is greater than one tenth of the wavelength, and it is for that range of periods that the wavelengths differ significantly. This observation agrees with that of Mindlin (1951), who reached the same conclusion on the basis of the wave velocities.

It is also worth noting here that a scale analysis of Equation (4) may be carried out, choosing physically reasonable ranges for its various parameters, and for the period and travelling wavelength. When this is done, it is found that the most significant terms in Equation (4) are precisely those of Equation (7), thus confirming the conclusions reached above. It was, however, necessary

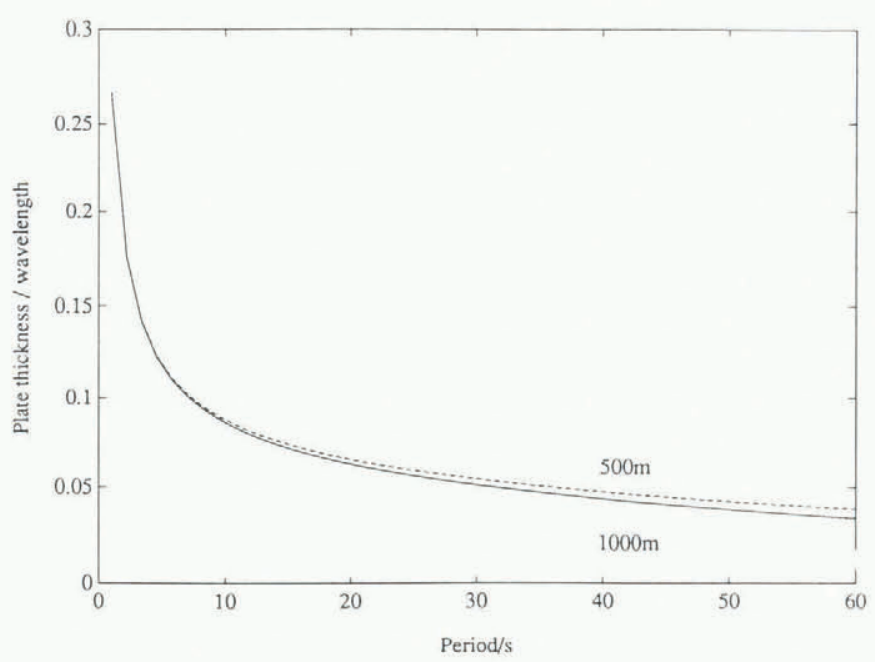

Fig. 4. Plate thickness divided by travelling wavelength as given by the thick-plate model plotted against period for $200 \mathrm{~m}$ thick ice on water of $1000 \mathrm{~m}$ depth and $500 \mathrm{~m}$ depth. 
Fox and Squire: Ocean and ice shelf coupling

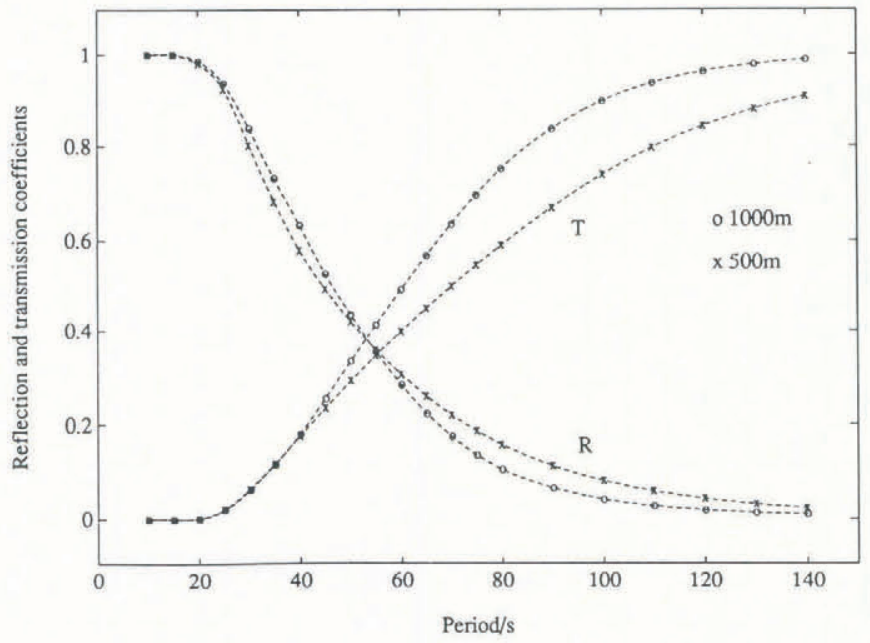

Fig. 5. Reflection and transmission coefficients plotted against period for $200 \mathrm{~m}$ thick ice on water of $1000 \mathrm{~m}$ and $500 \mathrm{~m}$ depth found using the thick-plate model.

to go through the full procedure because of the presence of the evanescent wave modes. These modes have wavelengths which approach zero as $n \rightarrow \infty$ and, hence, could be altered markedly by the additional terms of Equation (4). In the event they were not.

\section{Reflection and transmission characteristics}

The reflection and transmission coefficients for the amplitude of surface displacement, denoted $\mathcal{R}$ and $\mathcal{T}$ respectively, can be calculated from the coefficients of potential $I, R$ and $T$ using the following relationships

$$
\mathcal{R}=\left|\frac{R}{I}\right| \quad \text { and } \quad \mathcal{T}=\left|\frac{T}{I}\right| \frac{\kappa_{\mathrm{T}} \sinh \left(\kappa_{\mathrm{T}} H\right)}{k_{\mathrm{T}} \sinh \left(k_{\mathrm{T}} H\right)}
$$

These coefficients are plotted over the range of periods 10 to $140 \mathrm{~s}$ in Figure 5. Note that the transition from total reflection to total transmission occurs around a wave period of $60 \mathrm{~s}$. Incoming ocean waves with periods less than $20 \mathrm{~s}$ are completely reflected for both water depths, whereas those with periods somewhat longer than $140 \mathrm{~s}$ are fully transmitted in the $1000 \mathrm{~m}$ water depth case. Full transmission in the $500 \mathrm{~m}$ water depth case occurs at still longer periods. Note that the transmission coefficient is generally smaller when the water is $500 \mathrm{~m}$ deep as opposed to $1000 \mathrm{~m}$ deep. However, the reflection coefficient for $500 \mathrm{~m}$ deep water is appreciably larger than that for $1000 \mathrm{~m}$ deep water only for periods above $50 \mathrm{~s}$, whereas that relationship is reversed for periods below $50 \mathrm{~s}$. Essentially identical results are predicted when the thin-plate equations are employed.

\section{Ocean wave-induced strain in an ice shelf}

Measurements of flexural gravity waves at the surface of an ice shelf are more usually made in terms of the surface strain rather than the transverse displacement. The strain in a plate is simply half its thickness divided by the radius of curvature which, for small amplitude waves, reduces to

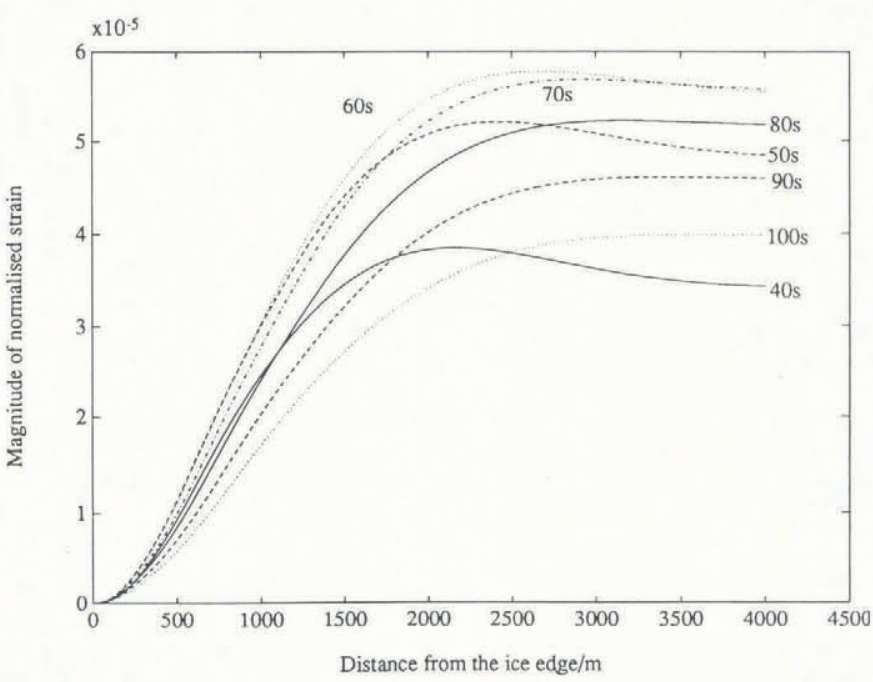

Fig. 6. Magnitude of strain at the surface of an ice shelf plotted against distance from the edge of the ice in metres for wave periods of $40,50,60,70,80,90$ and $100 \mathrm{~s}$. The geometry is $200 \mathrm{~m}$ thick ice on water of $1000 \mathrm{~m}$ depth and the incoming wave amplitude is $1 \mathrm{~m}$. The thick-plate model was used.

$$
\text { strain }=\frac{h}{2} \frac{\partial^{2} w}{\partial x^{2}}
$$

Thus, the strain is the product of the amplitude of a wave and the curvature produced by a unit amplitude wave of the same period. For a fixed wave height in the open sea, increasing period will increase the transmission coefficient and, hence, the amplitude at that period in the ice. At the same time, however, increasing period will decrease the curvature of a unit height wave. It is clear, therefore, that the maximum strain at the surface of the ice shelf will be generated, for fixed amplitude ocean waves, when the period is in the range between total reflection and total transmission.

The magnitude of strain generated by $1 \mathrm{~m}$ amplitude ocean waves with periods of 40 to $100 \mathrm{~s}$, in $10 \mathrm{~s}$ intervals, is plotted in Figures 6 and 7 for water depths of 1000

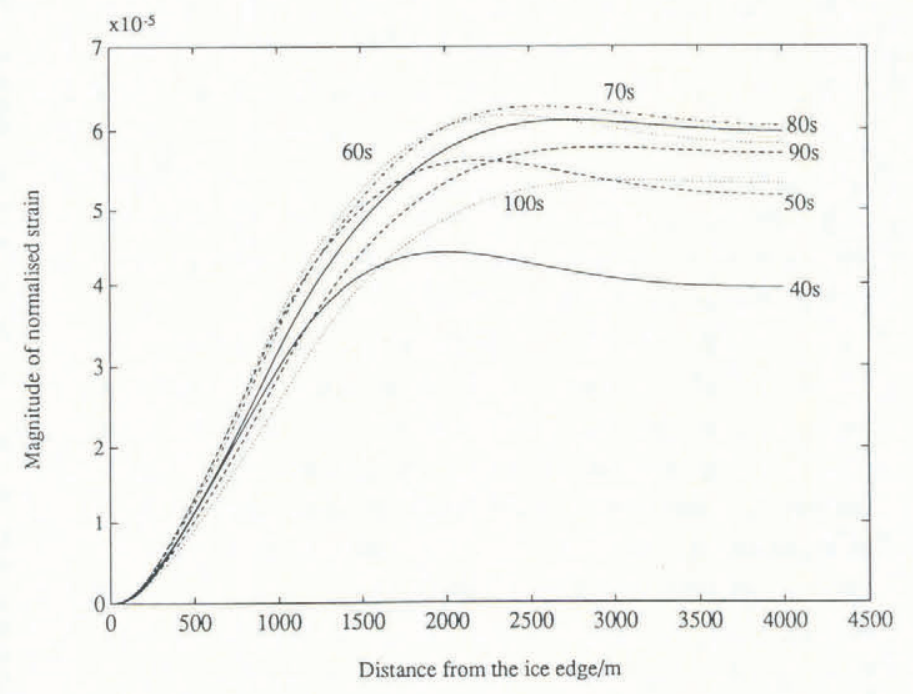

Fig. 7. Magnitude of strain for the same geometry and periods as Figure 6 except with $500 \mathrm{~m}$ deep water. 
Fox and Squire: Ocean and ice shelf coupling

and $500 \mathrm{~m}$, respectively, as a function of distance from the edge of the ice shelf. Each curve shows some characteristic features. In each case there is no strain at the ice edge: indeed, this is the boundary condition in Equation (10). After a transition, the magnitude of strain reaches a far field value, due solely to the transmitted wave. The strain in the intermediate range is affected by the strain due to the evanescent and damped travelling modes as well as the travelling wave. For some wave periods, e.g. $50 \mathrm{~s}$, the magnitude of strain reaches a peak, at an intermediate distance, which is greater than the far field value.

Note that the maximum magnitude of strain is exhibited for 60 and $70 \mathrm{~s}$ wave periods with 1000 and $500 \mathrm{~m}$ deep water, respectively. Indeed, for $1000 \mathrm{~m}$ deep water, the $60 \mathrm{~s}$ period generates the greatest strain for distances up to $3000 \mathrm{~m}$ from the edge and close to the greatest strain for greater distances. For $500 \mathrm{~m}$ deep water, waves with a $70 \mathrm{~s}$ period generate close to the greatest strain for distances up to $2000 \mathrm{~m}$ from the edge and the greatest strain for greater distances. This implies that the maximum coupling between ocean waves, or swell, and strain in the ice shelf occurs at $60 \mathrm{~s}$ for water depths of $1000 \mathrm{~m}$ and at $70 \mathrm{~s}$ for depths of $500 \mathrm{~m}$, at least for $200 \mathrm{~m}$ thick ice. It is interesting to note that the strain generated in $200 \mathrm{~m}$ thick ice, at each period, is greater for the shallower water geometry despite the fact that the transmission coefficient is lesser for that case. This is evidently due to the corresponding decrease in wavelength and, hence, increase in curvature of an ice-coupled wave, of a given period, when the water depth is decreased. This more than compensates for the decrease in transmission.

Figures 6 and 7 show that the strain in an ice shelf should be a sensitive detector of ocean waves with periods of $50-80 \mathrm{~s}$. There have been some measurements of long-period oscillations in an ice tongue against which we can calibrate the strains predicted in Figure 7. Whereas the geometry of an ice tongue does not conform exactly to the model analyzed, we use these measurements in the absence of more appropriate data. The authors would like to obtain more testing data against which the theory can be compared and would welcome input on this point. Propagating oscillations with periods of $40-50 \mathrm{~s}$ and with a strain amplitude of approximately $5 \times 10^{-8}$ were observed in the Erebus Glacier Tongue in the austral summer of 1989 (personal communication from W.H. Robinson). As a rough calculation, we can use the coupling calculated above to infer the size of seas required to drive such an oscillation. With reference to the curve for $50 \mathrm{~s}$ in Figure 7, we see that a $50 \mathrm{~s}$ ocean wave would only need an amplitude of about $1 \mathrm{~mm}$ to generate such a strain. Given the difficulty of measuring long-period ocean waves, it is not improbable that long-period ocean waves of this amplitude could exist, even though none have been reported. The greatest strain observed in the Erebus Glacier Tongue occurred the year before the observation above and was of the order of $5 \times 10^{-7}$ strain (personal communication from W.H. Robinson). Again on the basis of our crude comparison, this would imply that 50 s seas rarely go above $1 \mathrm{~cm}$ in amplitude.

The Gui and Squire (1989) analysis mentioned earlier reaches similar conclusions. It is likely that reality lies somewhere between the two extremes, with the strain field due to the ice-tongue oscillation being partitioned into a propagating component travelling up the tongue together with a standing vibration.

\section{CONCLUSIONS}

The results of this paper have implications for the range of periods within which we might expect to measure strain on an ice shelf, and the suitability of the thin-plate model for calculating that strain. Some conclusions are summarised below:

- The simpler thin-plate model coincides with the thickplate model for periods above $20 \mathrm{~s}$. Below that period both models predict total reflection of incoming waves. Hence, in terms of the reflection and transmission coefficients, the two models give essentially identical results for all periods and for the two geometries considered. This conclusion may well hold in general, that is, for a range of ice thicknesses and water depths.

- For the geometries modelled, the maximum coupling between ocean waves and strain in the ice occurs for a 60 to $70 \mathrm{~s}$ wave period. This is nearly true for all distances less than far field and in the far field. Further, the ice shelf acts as a band-pass filter, reacting most strongly to periods around 60 to $70 \mathrm{~s}$. It is likely all geometries react similarly, with a band-pass response to incoming wave periods.

- Ocean wave driving of long wave period strain in an ice shelf is a plausible mechanism. The comparisons made with existing data indicate that mean wave heights of $1 \mathrm{~mm}$ at a $50 \mathrm{~s}$ period would account for the observed strains.

\section{ACKNOWLEDGEMENTS}

The authors are indebted to the New Zealand Foundation for Research, Science, and Technology, the University of Otago and the New Zealand University Grants Committee for their continued support. Colin Fox was a University Grants Committee Post-Doctoral Research Fellow when this work was carried out. The helpful comments of the reviewers and editor are acknowledged.

\section{REFERENCES}

Evans, D.V. and T.V. Davies. 1968. Wave-ice interaction. Hoboken, NJ, Stevens Institute of Technology. Davidson Laboratory. (Report 1313.)

Fox, C. and V.A. Squire. 1990. Reflexion and transmission characteristics at the edge of shore fast sea ice. J. Geophys. Res., 95(C7), 11,629-11,639.

Fox, C. and V.A. Squire. 1991. Strain in shore fast ice due to incoming ocean waves and swell. J. Geophys. Res., 96(C3), 4531-4547.

Gill, P.E., W. Murray, and M.H. Wright. 1981. Practical optimization. London, Academic Press.

Gui, E.H. and V.A. Squire. 1989. Random vibration of floating ice tongues. Antarct. Sci., 1(2), 157-163.

Hildebrand, F.B. 1965. Methods of applied mathemat$i c s$. Second edition. Englewood Cliffs, NJ, 179-181.

Holdsworth, G. and R. Holdsworth. 1978. Erebus Glacier tongue movement. Antarct. J. U.S., 13(4), 61-63. 
Fox and Squire: Ocean and ice shelf coupling

Mindlin, R.D. 1951. Influence of rotary inertia and shear on flexural motions of isotropic, elastic plates. $J$. Appl. Math., 18, 31-38.

Phillips, O.M. 1977. The dynamics of the upper ocean. Second edition. Cambridge, etc., Cambridge University Press.

Squire, V.A. and C. Fox. 1990. The wave structure in the seas off an ice edge. In Murthy, T.K.S., J.G. Paren, W.M. Sackinger, and P. Wadhams, eds. The technol- ogy for polar operation. Proceedings of the Second International Conference on Ice Technology, held at Downing College, Cambridge University, UK, during 18-20 September 1990. Southampton and Boston, MA, Computational Mechanics Publications, 21-32.

The accuracy of references in the text and in this list is the responsibility of the authors, to whom queries should be addressed. 